[0212-7199 (2004) 21: 12; pp 607-610] ANALES DE MEDICINA INTERN Copyright (C) 2004 ARAN EDICIONES, S.L.

AN. MED. INTERNA (Madrid) Vol. 21, N. ${ }^{\circ} 12$, pp. 607-610, 2004

\section{Lo permanente y lo cambiante de la Medicina Interna: hacia una precisión de su especificidad}

\author{
R. BATALLER SIFRE \\ Coordinador Unidad de Medicina Interna. Departamento de Medicina. Universitat de \\ Valencia
}

WHAT IS PERMANENT AND CHANGING IN INTERNAL MEDICINE: TOWARD A BETTER UNDESTANDING OF ITS SPECIFICITY

Bataller Sifre R. Lo permanente y lo cambiante de la Medicina Interna: hacia una precisión de su especificidad. An Med Interna (Madrid) 2004; 21: 607-610.

Entrados ya en el siglo XXI, y tras una permanente -y a veces acelerada- metamorfosis de la Medicina Interna (MI) tanto de su aplicación clínica como de su inserción en los planes de enseñanza de Medicina, parece justificada una reflexión acerca de dicho proceso, por parte de todos, pero especialmente de los que hemos asistido y vivido un largo trecho de su tránsito, en particular en España.

\section{PERVIVENCIA DE LA MEDICINA INTERNA EN CUANTO TAL}

Apenas cuestionada en el ámbito de los denominados hospitales comarcales; en ellos mantiene la dimensión e influjo que antaño tuvo en los hospitales universitarios y los denominados de tercer nivel, en los que fraguaron y mantuvieron escuelas de enorme prestigio (recuérdese la Fundación Jiménez Díaz en Madrid y la Clínica Universitaria de Barcelona, bajo la égida de Pedro Pons y sucesores).

Con variaciones en su aplicación concreta, a partir de los años 60 se van configurando entre nosotros, tanto en la vertiente docente como asistencial, las denominadas especialidades médicas (cardiología, endocrinología, etc.), emanadas del tronco común de MI, aceptando la Administración las correspondientes materias tanto en las Facultades de Medicina como en los hospitales docentes, pero con apenas presencia de la MI en las áreas de Medicina Extrahospitalaria, a pesar de las recomendaciones y argumentos que se han ido esgrimiendo ante las autoridades (1). Sin embargo, es bien reciente la inclusión en algunos ámbitos de la figura del internista, como consultor, en Centros de Salud $(2,3)$, después de tantos años de espera.

Llegados al siglo XXI, en fin, la MI se encuentra asentada, en cuanto tal, como servicio clínico, en los hospitales clínicos de la Universidad, en los denominados de Tercer Nivel (¿no sería mejor cambiar esta denominación?), en los de ámbito provincial y en los comarcales en equilibrio, no siempre fácil, con la función y responsabilidades de las especialidades médicas derivadas de ella (y algo también con las de ámbito quirúrgico, con las traídas y llevadas "especialidades médico-quirúrgicas").

Por lo que respecta a la docencia universitaria, la evolución aún ha sido más drástica (4-6). Por un lado la denominación MI no existe en el nuevo plan de estudios del Estado Español (BOE 20 de noviembre de 1990).

Es curioso, empero, que haya sido muy fluido que siga habiendo la Unidad Docente de MI en distintas Facultades de Medicina/Departamentos de Medicina, con una tarea docente en unas ocasiones restringida a la patología general y en otras con un más amplio contenido (enfermedades infecciosas, toxicología clínica, enfermedades sistémicas, osteología clínica, etc.).

En ocasiones algunos hemos planteado que la Unidad Docente de MI convendría desglosarla en dos: a) Área de Patología General, ligada a hospitales pero más a la Medicina Extrahospitalaria (Centros de Salud); y b) Área de MI en cuanto tal, asentada en hospitales y, cada vez más, con una misión integradora de las especialidades derivadas de ella, en los centros de especialidades (CE), no sólo con carácter consultor, sino también con articulación espacial de sus actividades (Tabla I).

\section{EL ENTORNO DE LA MEDICINA INTERNA}

\section{LA MEDICINA GENERAL (MG), DE FAMILIA (MF) Y COMUNITARIA}

Aunque a nivel institucional (Servicio Nacional de Salud), los campos se encuentran deslindados (MG: Centros de Salud; MI: hospitales) hay una cercanía entre ambos ámbitos y, de

Trabajo aceptado: 8 de noviembre de 2004

Correspondencia: Ramón Bataller Sifre. Unidad de Medicina Interna. Departamento de Medicina. Universitat de Valencia. Avda. Blasco Ibáñez, 15. 46010 Valencia. e-mail: ramon.bataller@uv.es. 
TABLA I

MEDICINA INTERNA INTEGRADA (S. XXI)

\begin{tabular}{ll}
\hline Ámbito asistencial & Ámbito académico \\
\hline $\begin{array}{l}\text { Medicina Familiar y Comunitaria } \\
\text { (Centros de Salud y Unidades de }\end{array}$ & Unidad Docente Medicina Familiar y \\
Hospitalización a Domicilio) & (Asociada a Medicina Preventiva y Social) \\
Medicina Interna General & Unidad Docente de Patología General \\
(Centros de Especialidades) & (Síndromes, Semiología y Semiotecnia) \\
Medicina Interna & Unidad Docente de Medicina Interna \\
(Hospitales) & (Toxicología Clínica, Infecciosas, Sistémicas \\
& y Metabólicas; Módulo de Integración) \\
\hline
\end{tabular}

algún modo, cierto solapamiento de sus contenidos. Una parte de la formación del MF tiene lugar actualmente dentro de los Servicios de MI hospitalarios. Así entendemos que debería ocurrir también al revés, o sea que los residentes de MI rotaran algún tiempo por los Centros de Salud. Dicha vivencia formativa recíproca (y también posteriormente en la correspondiente formación continuada) estrecharía lazos entre los dos tipos de profesional: el Internista y el Generalista. En este sentido se viene manteniendo en el seno de las Sociedades Alemanas de MI y de MG, patrocinados por su Ministerio de Salud, encuentros para llegar a un consenso que tipifique bien el cometido respectivo, si bien, incluso, se viene postulando la posible materialización de una especialidad de MI y MG al lado de la MI clásica y de la MG y MF (7). Frecuentemente cierto grupo de enfermedades son, según el momento y grado de expresión y complejidad de las mismas, motivo de atención sucesiva por dichos dos grupos de especialistas (piénsese, entre otros, en el síndrome metabólico, la hipertensión arterial y la insuficiencia cardíaca). En España es escasa la atención que se presenta a esta cuestión, habida cuenta del "confinamiento" de la MI en los Hospitales, aunque hay balbuceos de atención al problema con inclusión de Internistas en Centros de Salud (CS), inicialmente como consultores (v. antes). La existencia de Servicios de MI en los CS y CE supondría un gran ahorro de gastos; ignoramos por qué la administración no completa y amplia este criterio, compartido por muchos (8). En algunos Centros Médicos Universitarios se lleva a cabo una cooperación intradisciplinar entre MI General (MIG), MF y Pediatría (9).

La integración de la MF ("Primaria") en el actual Plan de Estudios lo es a nivel optativo (no troncal); como decíamos antes, la MI no figura en cuanto tal sino dividida en las asignaturas que integran troncalmente el apartado de "Medicina y Cirugía de Aparatos y Sistemas" y en la materia de "Patología General". Es curioso que, a la hora de las prácticas de la Licenciatura, en especial para alumnos de sistema Erasmus, se recurre a los Servicios Hospitalarios de MI, tras dejarla sin contenido específico en el marco de la enseñanza teórica. Esta incongruencia supone un perjuicio real para los estudiantes y deja perplejo al profesorado de MI.

\section{LAS ESPECIALIDADES Y CAMPOS MÉDICOS DERIVADOS Y VECINOS DE LA MI, PEDIATRÍA Y GERIATRÍA INCLUIDAS}

La segregación clásica de la Pediatría del tronco general en la Medicina (desde principios del siglo XIX) (10), indis- cutida en general, aunque asentada, más que en criterios fisiopatológicos, en los de tipo cronológico (edad) y social (Puericultura), puede servir de punto de comparación con similares criterios para fundamentar la Geriatría, que, de asentarse, dejaría para la MI la denominada por algunos "Medicina del Adulto". De un modo o de otro la Geriatría envuelve o, mejor, integra buena parte de la MI actual; el aumento de la vida media entre nosotros supone un gran desplazamiento de la MI hacia la Geriatría. Argumentos hay a favor (11) y otros en contra, pero el troceamiento de lo que queda de la MI también por cuestiones más cronológicas (edad) o de otra índole (Gerocultura) supondría una escisión como la que representó en su momento la de la Pediatría.

Mención especial merecería la denominada "Medicina de la mujer", curiosamente asociada alrededor de un fenómeno fisiológico como es la menopausia; su carácter inconsistente no augura sino una corta sobrevida a este periplo paramédico.

Quedan ya lejanos los años en que las especialidades médicas se entendían como avaladas con y para el desarrollo y aplicación de técnicas complejas (12), como "instrumento consultivo de ayuda para el médico generalista" (13); el 95\% de pósters presentados al Congreso 109 (2003) de la Sociedad Alemana de MI se adscribieron a las correspondientes especialidades (Endocrinología, Cardiología, Oncohematología, etc.); tan solo el $5 \%$ pertenecían al grupo "misceláneo"; ¿cómo inscribir en la MI tradicional especialidades que tienen su propia sociedad científica?; ¿qué queda de "específico" de la MI, que no haya sido tomado por las correspondientes especialidades derivadas de ella?. Entendemos que ciertos trastornos requieren atención "general" más ancha, tal como las enfermedades infecciosas, las intoxicaciones, las enfermedades inmunes sistémicas y las metabólicas; pero aunque las molestias concretas (cefalea, precordialgia, vómitos, etc.) induzcan al enfermo, y a veces a su MF, a solicitar el concurso del especialista correspondiente (neurólogo, cardiólogo, digestólogo, etc.) el paciente sigue siendo uno y la afección preferencial o más destacada que tiene implica o se expresa también, en mayor o menor medida, en los demás territorios de su organismo; en efecto, la Asociación Europea de Médicos Especialistas entiende que la MI supone la "práctica asistencial a nivel de individuo y no de órgano" (14).

De ahí la necesidad actual de una Integración de las Especialidades derivadas de la MI, superando en buena medida la problemática de las Especialidades Médicas cuyo número se sigue ensanchando; se trataría de un cierto papel de síntesis que le corresponde a la MI moderar (15), pasando de alguna manera a ser la Especialidad de la Integración (16).

En la atención y promoción de nuevos campos de especialización y de enfermedades emergentes y/o de prevalencia creciente en cada área geográfica (VIH, enfermedades autoinmunes, intoxicaciones crónicas, miopatías, etc.), se van articulando "Unidades", que progresivamente desembocan en Servicios Hospitalarios y Docentes, según ámbitos y conjunturas. Actuaría la MI, en fin, de origen y desarrollo de una serie de campos médicos nuevos, al modo de las "stem cells", de un modo casi que inagotable con el tiempo, hasta un nivel de maduración que conduciría al nacimiento de una nueva especialidad, como ha sido el caso durante décadas, sobretodo a partir de los años 60 (v. antes). 
LA PROPIA DENOMINACIÓN, CONTENIDO Y APLICACIÓN DE LA MEDICINA INTERNA

A pesar de la intensa e inacabada segmentación de su contenido en Especialidades, la MI sigue bien asentada en el mundo sanitario, pero tan poco entendida como siempre por el público en general, y es que a los que nos dedicamos a su cultivo sigue resultándonos difícil, cuando no enojoso, explicar a terceros no médicos el significado de su denominación, en verdad anclado en tiempo pasado cuando se distinguía entre MI y externa (Cirugía), diferenciación que se ha ido desdibujando con el tiempo y de manera acelerada con el progreso de las endoscopia y de los métodos de imagen (ecografía, TAC, RNM, etc.), hasta el punto de no poder caracterizarla actualmente ya por una cuestión espacial ("Interna”) (17).

La denominación MI General (MIG) ha ido tomando cuerpo en estos últimos años, en especial en USA, contraponiéndola a la propia MI sin más (18), que estaría representada e inmersa en las Especialidades Médicas (allí denominadas Subespecialidades) $(8,19)$, si bien que fragmentariamente, hurtándole a la propia MI el carácter ancho, holístico que le corresponde; en efecto, London (20), en el IX Congreso Mundial de MI, entiende que el Internista "trata de comprender la enfermedad desde su nivel molecular, subcelular, de órgano, de organismo en su conjunto y de amplios grupos sociales, incluyendo la antropología social".

En algunos Hospitales Universitarios de otros países europeos ya se ha asumido aquella denominación (MIG) y no en los homólogos de España.

Se viene asimilando también la MI con la denominada "Medicina del Adulto" segregándola de la Pediatría y de la Geriatría, aunque aquella denominación no ha cuajado entre nosotros afortunadamente (v. antes).

¿Cuál (o cuáles) serían los criterios actuales que estarían -que están- en la base de sustentación de esta materia (por no llamarla especialidad como a sus "subespecialidades" derivadas), que justifican la pervivencia de la MI en nuestra sociedad y su sistema sanitario?

Las claves podrían ser:

- La tendencia, de entrada, a una consideración médica "de cabeza a pies" de quien nos consulta por el motivo o por las molestias que fuere; bien es verdad que numerosos cultivadores de las especialidades derivadas de la MI, en mayor o menor medida, contemplan -pero quizás no llevan a la práctica tanto- una consideración de dicha naturaleza. Una cierta propensión a detectar correlaciones entre aparatos y sistemas y al análisis de las mismas, entraría a formar parte del núcleo operativo y hasta disposicional del internista (averiguar la posible patología tiroidea en una gastritis crónica, o la alteración glomerular en una hepatitis crónica, o el posible origen autoinmune sistémico de una pleuresía, etc., serían algunos ejemplos de la postura más acendrada a dicho respecto del internista que de otros especialistas), buscando, en fin, lo que Portugal Alvarez llama (21) "manifestaciones satélites" de las enfermedades internas.

- La dedicación en tiempo a integrar todos los datos, a veces numerosos, que nos aporta el paciente y su familia, desde que su problema clínico se empezó a atender (MF, Compañías de Seguros, laboratorios de Bioquímica Clínica y otros, medicación previa recibida y tolerancia y/o efectos de la misma, informes médicos previos de otros especialistas, etc), labor esta a menudo ardua y trabajosa, que precisa de tiempo disponible (hoy por hoy posible solo, y no siempre, en el Hospital, ya sea en Consultas Externas o en Salas de Hospitalización, que no en los CE ni CS); de algún modo, el Internista, junto con MG o MF tendría, entre otras, esta misión de "hilvanador", "integrador" de "cabos dispersos", que por separado no aportan solución al problema clínico pero que conjuntados, a modo de articulación de las piezas de un puzzle, pueden suponer un "centrado" del diagnóstico, en muchas ocasiones pluriorgánico y/o sistémico. En este ámbito, la frecuente plurifarmacoterapia habría de ser, y es a menudo, una tarea de análisis, enraizada con la toxicología clínica, en cuanto materia de estudio incardinada en el nuevo plan de estudios de medicina inaugurado hace una década en nuestras facultades (uno de los aciertos que le encontramos).

- Su marcado cometido diagnóstico que comporta el manejo de fuentes (22-28) que posibilitan la adecuada filiación, no solo sindrómica, sino también clínico-topográfica y etiológica del proceso que se atienda.

- La detección temprana sagaz de neoplasias únicas (y a veces sincrónicas), que no siempre se expresan inicialmente con la clínica y semiología propia del órgano primariamente afecto (síndromes paraneoplásicos).

- La dedicación de un tiempo a las dudas de orden laboral, social, nutricional y otros en que se halla inmerso el paciente y la familia que lo atiende como componente necesario para que la suma de distintos dictámenes médicos encuentre la coordinación adecuada, como base para una mayor eficacia de las distintas medidas terapéuticas (algo así como la "cultura" del adulto afín a la gerocultura del anciano).

- Dada la anchura del campo de la MI, la necesidad por lo general, en el orden de la Investigación Clínica, de que el Internista opte por campos más recortados (HTA, osteoporosis, lípidos, insuficiencia cardíaca, etc.) que los que suelen abordar los especialistas de cada órgano o sistema (Cardiología, Digestivo, etc.) y así, por ejemplo, en la Sociedad Española de MI hay articulados grupos de trabajo de campos concretos como los mencionados y otros (osteoporosis, inflamación, insuficiencia cardíaca, etc.).

-En el terreno de la docencia, su papel hay que catapultarlo, afirmándose, al menos, en tres órdenes de dedicación:

- Contenido de la Patología General en especial lo relativo a la semiología, síndromes y semiotecnia, así como aspectos de la fisiopatología que correlacionan órganos y sistemas, en concreto el sistema nervioso, hormonal e inmunitario $(29,30)$.

- Afecciones que por su propia naturaleza implicarían ampliamente al organismo (infecciones, intoxicaciones, enfermedades sistémicas y metabólicas).

- Quizás también, las afecciones de más de un órgano, no a nivel de repercusión, sino de entrada (p. ej. riñón-hígadocerebro en la enfermedad de Wilson, páncreas-hígado-endocrinas en la hemocromatosis, etc.).

- Como módulo de "integración" abordando áreas o aspectos de cada especialidad que constituyan nexo con los trastornos generales (p. ej. cefaleas en Neurología, insuficiencia cardíaca en Cardiología, osteoporosis en Reumatología, etc.).

\section{AGRADECIMIENTO}

A María Dolores Alberola García por la ordenación y puesta a punto del texto. 


\section{Bibliografía}

1. Freytro JE. Papel de los internistas en los Centros de Salud. XVIII Congreso Sociedad Española de Medicina Interna. Alicante. 1988.

2. Pujol Farriols R, Corbella Viros X. El Especialista en Medicina Interna como consultor de Atención Primaria. Med Clin (Barc) 2004; 122: 60-1

3. Grupo para la Asistencia Médica Integrada y Continua de Cádiz. La consultoría en Medicina Interna en Atención Primaria mejora la eficacia de la atención médica. Med Clín (Barc) 2004; 122: 46-52.

4. De Portugal Alvarez J. Una asignatura en crisis. An Med Interna (Madrid) 1996; 13:299-302.

5. Rozman C. Reflexiones sobre la Medicina Interna en la Universidad. Med Clin (Barc) 1981; 76: 193-202.

6. Ortiz Vázquez J. Presente y futuro de la Medicina Interna: la Medicina Interna como Especialidad, como Departamento Hospitalario y como Enseñanza. An Med Interna (Madrid) 1984; 1: 100-107.

7. Schuster HP. Deustsche Gesellchaft für Innere Medizin, Bundesverband Deutscher Internisten und die Beschlüsse von Rostock. Med Klin 2002; 97:572.

8. Kellet J, Hillen H. Introduction to Internal Medicine. Internist 2003; 44: M 170-6.

9. Sherger JE, Rucker LL, Morrison EH, Cygan RW, Hubbel A. The Primary Care Specialities working together: a model of success in a academic environment. Academic Medicine 2000; 75: 693-8

10. Lain Entralgo P. Historia de la Medicina Moderna y Contemporánea. Científico-Médica. Barcelona 1963. p. 428.

11. Hazzard WR. The Department of Internal Medicine: Hub of the Academic Health Center response to de aging imperative. Ann Intern Med 2000; 133: 293-6.

12. Beeson P B. One hundred years of American Internal Medicine. Ann Intern Med 1986; 105: 436-44.

13. Petersdorf R. El imperativo generalista. Hosp Pract (Ed esp) 1994; 9: 56

14. Garrido Peralta M. La Medicina Interna y el Mercado Común Europeo. Medicamenta 1996; 27: 233-6.
15. Stahl J. La mission de synthése de la Medicine Interne, en Integration in Internal Medicine, Ed Dunning AJ. Excerpta. Amsterdam 1997. p. 3-12.

16. Peláez Redondo J. La enseñanza de la medicina en el Post-Grado An Med Interna (Madrid) 1983; 1: 75-78

17. Lain Entralgo P. La situación actual de la Medicina Interna como problema histórico. VIII Reunión de la Sociedad Española de Medicina Interna. Madrid 1969.

18. Fetscher S. Neuere Entwicklungen im Verhältnis von Internist und Allgemeinarzt in dem USA. Internist 1998, 3: M61-71.

19. Earley LE. Alternative Pathways for Training the General Internist and the Medical Subspecialist. Ann Intern Med 1992; 116: 1080-3.

20. London FM. Internal Medicine, change and challenge, en Integration in Internal Medicine. Ed. Dunning AJ. Excerpta Medica. Amsterdam, 1967. p. 540-5.

21. Portugal Alvarez J. Manifestaciones satélites de las enfermedades internas. Masson. Barcelona 1994.

22. Jiménez Diaz C, Jiménez Casado M, Rozman Borstnar C. Diagnóstico Diferencial. Daimon. Madrid 1963.

23. Marañon Posadillo G. Manual de Diagnóstico Etiológico. Espasa Calpe. Madrid 1954

24. Ortner N. Sintomatología Clínica de las Enfermedades Internas. Usón. Barcelona 1926.

25. Kaufmann W. Internistische Differentialdiagnostik. Schattauer. Stuttgart 1992.

26. Siegenthaler W. Differentialdiagnose Innerer Krankheiten. Thieme. Stuttgart 2000.

27. Green HL, Johnson WP, Maricic JM. Toma de Decisiones en Medicina Mosby. Madrid 1995.

28. Greene HC, Glassock RJ, Kelley MA. Introduction to Clinical Medicine. Decker. Filadelfia 1991.

29. Guyton AC, Hall J E. Manual de Fisiología Médica Interamericana. Madrid 2001. p. 5 .

30. Mösinger M. Neuro-Endocrinología y Neuro-Ergonología. Noguer. Barcelona 1957. 